\title{
NORMAL FORMS IN PARTIAL MODAL LOGIC
}

\author{
JAN O. M. JASPARS \\ Institute for Language Technology \& AI, Tilburg University \\ P.O. Box 90153, 5000 LE Tilburg, The Netherlands \\ E-mail: JASPARS@KUB.NL
}

\begin{abstract}
A "partial" generalization of Fine's definition [Fin] of normal forms in normal minimal modal logic is given. This means quick access to complete axiomatizations and decidability proofs for partial modal logic [Thi].
\end{abstract}

Introduction. From the viewpoint of formal linguistics, cognitive science and artificial intelligence, there appears to be a natural interest in the combinations of partiality and modality $[\mathrm{BaP}][\mathrm{Kam}][\mathrm{FaH}]$. Modal operators cover the uncertain status of the informational content of propositional attitudes. Partiality is the formal translation of the incomplete behavior of these attitudes. The sources of uncertainty, multiple possible worlds (interpretations), do not have a two-valued character such as in classical modal logic. We take these possible worlds to be partial. They do not necessarily assign a Boolean truth value to all propositions.

In this paper we will define normal forms for partial modal logic, which is induced by a "partial" generalization of Fine's definition of normal forms in classical (total) modal logic [Fin]. It turns out that these normal forms for partial modal logic are more difficult to handle in proving that all formulae are equivalent to a disjunction of normal forms. This bad behavior can be explained by taking normal forms to be full modal descriptions of worlds. In total possible world semantics mutually coherent normal forms must be the same for this reason. In the partial semantics this is not the case, and therefore the conjunction of two different normal forms does not guarantee inconsistency. In partial propositional logic it is guaranteed that two coherent normal forms produce a new unique normal

1991 Mathematics Subject Classification: 03B45.

This research is supported by the programme "Dialogue management and knowledge acquisition" (DenK) of the Tilburg-Eindhoven Organization for Inter-University Cooperation (SOBU).

The paper is in final form and no version of it will be published elsewhere. 
form, but in the modal case this is certainly not true, as we will point out in the paper. We can prove, however, that the conjunction of two normal forms has the weaker disjunction property stated above, which turns out to suffice for proving the disjunction property for the full language that we will use.

This disjunction property will give us the completeness and decidability result for partial modal logic immediately, just as for normal modal logic in [Fin].

The following definition gives us the language, $\mathcal{L}^{\square}$, that we will use.

Definition 1. $\mathcal{L}^{\square}$ is the smallest set such that

- $\mathbb{P} \subset \mathcal{L}^{\square}$ where $\mathbb{P}$ is a finite set of primitive propositions $(\mathbb{P}=\{p, \ldots, q\})$;

- $\perp \in \mathcal{L}^{\square}$;

- $\varphi, \psi \in \mathcal{L}^{\square} \Rightarrow \neg \varphi \in \mathcal{L}^{\square}, \varphi \wedge \psi \in \mathcal{L}^{\square}, \square \psi \in \mathcal{L}^{\square}$.

The members of $\mathcal{L}^{\square}$ are called formulae. The modal depth of a formula $\varphi, m \varphi$, is determined by the following recursive definition: $\mathrm{m} p=\mathrm{m} \perp=0, \mathfrak{m} \neg \varphi=\mathfrak{m} \varphi$, $\mathrm{m} \varphi \wedge \psi=\max \{\mathrm{m} \varphi, \mathrm{m} \psi\}, \mathrm{m} \square \varphi=\mathrm{m} \varphi+1 . \mathcal{L}_{n}^{\square}$ denotes $\left\{\varphi \in \mathcal{L}^{\square} \mid \mathrm{m} \varphi \leq n\right\}$.

Partial possible worlds. Partial modal logic is the natural logic which arises from "partializing" standard Kripke models for modal logic.

Definition 2. Let $F=\langle W, R\rangle$ be a Kripke frame, that is, $W$ is a nonempty set, and $R$ is a binary relation on $W(R \subseteq W \times W)$. A partial Kripke model $M=\langle W, R, V\rangle$ on $F$ is the result of adding a partial valuation $V$ to the worlds appearing in $W$. Formally, $V: W \times \mathbb{P} \rightsquigarrow\{0,1\}\left(^{1}\right)$. The truth/falsity conditions are now fully determined by the following recursive composition. Let $w \in W$.

$$
\begin{array}{ll}
M, w \models p \text { iff } V(w, p)=1 \text { for all } p \in \mathbb{P}, & M, w=p \text { iff } V(w, p)=0 \text { for all } p \in \mathbb{P}, \\
M, w \vDash \perp, & M, w=\perp, \\
M, w \models \neg \varphi \text { iff } M, w \neq \varphi, & M, w=\neg \varphi \text { iff } M, w \models \varphi, \\
M, w \models \varphi \wedge \psi \text { iff } M, w \models \varphi \& M, w \models \psi, & M, w \neq \varphi \wedge \psi \text { iff } M, w \neq \varphi \text { or } M, w \neq \psi, \\
M, w \models \square \varphi \text { iff } \forall v(R(w, v) \Rightarrow M, v \models \varphi), & M, w \neq \square \varphi \text { iff } \exists v(R(w, v) \& M, v \neq \varphi) .
\end{array}
$$

Furthermore, $\psi$ is said to be a consequence of $\varphi$, denoted by $\varphi \models \psi$, if every pair $M, w$ that supports $\varphi(M, w \models \varphi)$ also supports $\psi$.

O bs ervation 1 . The definition of consequence does not behave totally contrapositional. Note that $p \wedge \neg p \models q$ but $\neg q \not \models \neg(p \wedge \neg p)$. The most important shortage of partial logic is of course the omission of the law of the excluded middle; the middle (undefined) is no longer excluded: $M, w \not \models \varphi$ does not entail $M, w \models \neg \varphi$.

$\left({ }^{1}\right) \rightsquigarrow$ means that $V$ is partial, that is, not defined for all the inputs on $V$ (the pairs of $W \times \mathbb{P})$. 
Axiomatizing partial Kripke models. In [Thi] it has been stated and proved that the axiom-schema below, which is called $\mathbf{M r L}+\left({ }^{2}\right)$, is complete and sound with respect to partial Kripke models. In this article the completeness proof procedure follows the ordinary Henkin procedure. The "partialization" is induced by generalizing the notion of maximal consistent sets to socalled consistent saturated sets, which are sets of formulae that are deductively closed, consistent and that obey the so-called disjunction property: if a disjunctive formula is a member of this set, then the membership of at least one of the disjuncts is guaranteed. We will follow Fine's normal form method [Fin], as was stated in the introduction. Both methods are still very close. A normal form can be interpreted as a unique representation of a consistent saturated set.

The following definition pictures the system $\mathrm{MrL}+$.

Definition 3. Let $\varphi \vdash \psi$ denote that $\psi$ is derivable from $\varphi$. By $\varphi \equiv \psi$ we abbreviate the relation of derivability in both directions. $\vdash_{\mathrm{cp}}$ abbreviates $\vdash$ and its contraposition: $\varphi \vdash_{\mathrm{cp}} \psi \Leftrightarrow \varphi \vdash \psi \& \neg \psi \vdash \neg \varphi$. $\equiv_{\mathrm{cp}}$ abbreviates the corresponding equivalence relation.

$$
\begin{aligned}
& \perp \vdash_{\mathrm{cp}} \varphi, \\
& \varphi \vdash \varphi, \\
& \neg \neg \varphi \equiv \varphi, \\
& \varphi \wedge \psi \vdash_{\mathrm{cp}} \varphi, \\
& \varphi \wedge \psi \vdash_{\mathrm{cp}} \psi, \\
& \varphi \wedge \neg\left(\wedge \chi \equiv_{\mathrm{cp}} \neg(\neg(\varphi \wedge \neg \psi) \wedge \neg(\varphi \wedge \neg \chi)),\right. \\
& \varphi \wedge \neg \varphi \vdash \perp, \\
& \varphi \vdash \psi \& \psi \vdash \chi \Rightarrow \varphi \vdash \chi, \\
& \varphi \vdash \psi \& \varphi \vdash \chi \Rightarrow \varphi \vdash \psi \wedge \chi, \\
& \varphi \vdash \psi \& \chi \vdash \psi \Rightarrow \neg(\neg \varphi \wedge \neg \chi) \vdash \psi, \\
& \square(\varphi \wedge \psi) \equiv_{\mathrm{cp}} \square \varphi \wedge \square \psi, \\
& \square\left(\neg(\varphi \wedge \psi) \vdash_{\mathrm{cp}} \neg(\square \varphi \wedge \neg \square \neg \psi),\right. \\
& \neg \square \neg(\varphi \wedge \neg \varphi) \vdash \perp, \\
& \varphi \vdash \psi \Rightarrow \square \varphi \vdash \square \psi \& \neg \square \neg \varphi \vdash \neg \square \neg \psi, \\
& \neg \perp \vdash \varphi \Rightarrow \neg \perp \vdash \square \varphi .
\end{aligned}
$$

Instead of $\neg \perp \vdash \varphi$ we also write $\vdash \varphi$, and call $\varphi$ a theorem of $\mathbf{M r L}+$. Note that the class of theorems in $\mathrm{MrL}+$ is very restricted and trivial.

In the rest of the paper we will abbreviate $\neg \perp, \neg(\neg \varphi \wedge \neg \psi)$ and $\neg \square \neg \varphi$ by $T$, $\varphi \vee \psi$ and $\diamond \varphi$ respectively.

$\left({ }^{2}\right)$ The logic is somewhat different here. In [Thi] $\perp$ does not appear. 
Observation 2. We state the following important principles that hold in $\mathrm{MrL}+$.

De Morgan laws:

$$
\neg(\varphi \wedge \psi) \equiv \neg \varphi \vee \neg \psi \quad \text { and } \quad \neg(\varphi \vee \psi) \equiv \neg \varphi \wedge \neg \psi .
$$

Distribution of $\wedge$ over $\vee$, and vice versa:

$$
\varphi \vee(\psi \wedge \chi) \equiv(\varphi \vee \psi) \wedge(\varphi \vee \chi) \text { and } \varphi \wedge(\psi \vee \chi) \equiv(\varphi \wedge \psi) \vee(\varphi \wedge \chi)
$$

Idempotency of $\wedge$ and $\vee$ :

$$
\varphi \equiv \varphi \wedge \varphi \text { and } \varphi \vee \varphi \equiv \varphi
$$

Commutativity:

$$
\varphi \wedge \psi \equiv \psi \wedge \varphi \quad \text { and } \quad \varphi \vee \psi \equiv \psi \vee \varphi
$$

Associativity:

$$
\varphi \wedge(\psi \wedge \chi) \equiv(\varphi \wedge \psi) \wedge \chi \quad \text { and } \quad \varphi \vee(\psi \vee \chi) \equiv(\varphi \vee \psi) \vee \chi
$$

Absorption:

$$
\varphi \vdash \psi \quad \text { iff } \quad \varphi \equiv \varphi \wedge \psi \text { and } \psi \equiv \varphi \vee \psi
$$

Distribution of $\diamond$ over $\vee$ :

$$
\diamond(\varphi \vee \psi) \equiv \diamond \varphi \vee \diamond \psi
$$

$\square / \diamond$ inter-relation:

$$
\square(\varphi \vee \psi) \vdash \square \varphi \vee \diamond \psi \& \square \varphi \wedge \diamond \psi \vdash \diamond(\varphi \wedge \psi) .
$$

The soundness of $\mathbf{M r L}+$ is straightforward, and is therefore left (to the reader).

Normal forms. The formalization of inference above gives us the syntactic equipment for implementing the concept of normal form. Before introducing this notion we first define some more syntactic abbreviation.

Let $\Phi=\left\{\varphi_{1}, \ldots, \varphi_{n}\right\}$ be a set of formulae. By $\bigwedge \Phi$ we mean the formula $\varphi_{1} \wedge \ldots \wedge \varphi_{n}\left({ }^{3}\right)$, and $\bigvee \Phi$ denotes $\varphi_{1} \vee \ldots \vee \varphi_{n}$. In case of $\Phi=\emptyset, \wedge \Phi:=\top$ and $\bigvee \Phi:=\perp$. By $\neg \Phi$ we mean the set $\left\{\neg \varphi_{1}, \ldots, \neg \varphi_{n}\right\}$. Analogously, $\square \Phi=$ $\left\{\square \varphi_{1}, \ldots, \square \varphi_{n}\right\}$ and $\diamond \Phi=\left\{\diamond \varphi_{1}, \ldots, \diamond \varphi_{n}\right\}$. Moreover, $\Phi \wedge \Psi:=\{\varphi \wedge \psi \mid \varphi \in \Phi$, $\psi \in \Psi\}$. We take the $\bigwedge$ and $\bigvee$ to be dominant over the ordinary connectives. So, for example,

$$
\bigwedge \neg \square \Phi \vee \diamond \bigvee \Phi=\left(\neg \square \varphi_{1} \wedge \ldots \wedge \neg \square \varphi_{n}\right) \vee \diamond\left(\varphi_{1} \vee \ldots \vee \varphi_{n}\right)
$$

We will also use indexed disjunctions and conjunctions:

$$
\bigvee_{i \in I} \varphi_{i}=\bigvee\left\{\varphi_{i} \mid i \in I\right\}, \quad \bigwedge_{i \in I} \varphi_{i}=\bigwedge\left\{\varphi_{i} \mid i \in I\right\}
$$

$\left({ }^{3}\right)$ Associativity legalizes the omission of parentheses. Associativity, commutativity and idempotence makes $\bigwedge \Phi$ unambiguous (modulo $\equiv$ ). It would be neater if we also ordered formulae lexicographically, but we expect the reader to be permissive on this point. 
Definition 4. The set $\mathcal{N}_{i}$ of normal forms of degree $i(i \in \mathbb{N})$ is determined by the following induction:

$$
\begin{aligned}
& \mathcal{N}_{0}=\{\bigwedge P \wedge \bigwedge \neg Q \mid P \subseteq \mathbb{P}, Q \subseteq \mathbb{P}, P \cap Q=\emptyset\}, \\
& \mathcal{N}_{n}=\left\{\bigwedge \diamond \Phi \wedge \square \bigvee \Phi \wedge \pi \mid \Phi \subseteq \mathcal{N}_{n-1}, \pi \in \mathcal{N}_{0}\right\} .
\end{aligned}
$$

The pair $[\pi, \Phi]$ with $\pi \in \mathcal{N}_{0}$ and $\Phi \subseteq \mathcal{N}_{n-1}$ abbreviates the normal form $\Lambda \diamond \Phi \wedge$ $\square \bigvee \Phi \wedge \pi\left({ }^{4}\right)$. A normal form of degree $0, \pi=\wedge P \wedge \wedge \neg Q$, will also be referred to as the pair $\langle P, Q\rangle$. Instead of $[\top, \Phi](=[\langle\emptyset, \emptyset\rangle, \Phi])$ we will also use $[\Phi]$.

This definition is slightly different from Fine's [Fin]. In this article the $\square-$ conjunct is replaced by a set of $\neg \diamond$-conjuncts. In total possible world semantics after summing up all possible (accessible) world descriptions, the rest of the world descriptions are not possible $(\neg \diamond)$. Partial possible world semantics lacks this comfort. As was stated in the introduction this is a technical disadvantage for proving that disjunctions over normal forms cover the full language (modulo $\equiv$ ).

Firstly, we show that normal forms, as defined above, are really full descriptions of partial worlds. A partial world corresponds to a normal form which uniquely generates the full theory of this world. This correspondence is embodied by a sequence of functions $\left\{\theta_{i}\right\}_{i \in \mathbb{N}}: \mathcal{W} \rightarrow \mathcal{N}_{i}$, where $\mathcal{W}$ denotes all pairs $M, w$ with $M=\langle W, R, V\rangle$ being a partial Kripke model and $w$ a world in $M$. The definition of this sequence runs as follows:

$$
\theta_{0}(M, w)=\bigwedge w^{+} \wedge \bigwedge \neg w^{-},
$$

where $w^{+}=\{p \in \mathbb{P} \mid V(w, p)=1\}$ and $w^{-}=\{p \in \mathbb{P} \mid V(w, p)=0\}$, and

$$
\theta_{n}(M, w)=\left[\theta_{0}(M, w),\left\{\theta_{n-1}(M, v) \mid R(w, v)\right\}\right] .
$$

THEOREM 1. Let $\varphi$ be a formula with modal depth at least as small as $n$ $\left(\in \mathcal{L}_{n}^{\square}\right)$. Then

$$
\begin{aligned}
& M, w \vDash \varphi \Leftrightarrow \theta_{n}(M, w) \vdash \varphi, \\
& M, w=\varphi \Leftrightarrow \theta_{n}(M, w) \vdash \neg \varphi .
\end{aligned}
$$

Proof. The $\Leftarrow$-direction is by soundness of $\mathbf{M r L}+$ and the observation that $M, w \models \theta_{n}(M, w)$.

The $\Rightarrow$-proof runs via induction on the construction of formulae, where only the $\square$-step deserves exposition.

$M, w \vDash \square \varphi \Rightarrow \forall v: R(w, v) \Rightarrow M, v \vDash \varphi \Rightarrow \theta_{n-1}(M, v) \vdash \varphi$ for all $v$ such that $R(w, v) \Rightarrow \bigvee \Theta_{n-1} \vdash \varphi$ with $\Theta_{n-1}=\left\{\theta_{n-1}(M, v) \mid R(w, v)\right\} \Rightarrow \square \bigvee \Theta_{n-1} \vdash \square \varphi \Rightarrow$ $\theta_{n}(M, w) \vdash \square \varphi$.

$M, w \neq \square \varphi \Rightarrow \exists v: R(w, v) \& M, v \neq \varphi \Leftrightarrow \theta_{n-1} \vdash \neg \varphi$ for certain $\theta_{n-1} \in \Theta_{n-1}$ $=\left\{\theta_{n-1}(M, v) \mid R(w, v)\right\} \Rightarrow \diamond \theta_{n-1} \vdash \neg \square \varphi$, and so $\theta_{n}(M, w) \vdash \neg \square \varphi$.

$\left({ }^{4}\right) \diamond \varphi_{1} \wedge \ldots \wedge \diamond \varphi_{n} \wedge \square\left(\varphi_{1} \vee \ldots \vee \varphi_{n}\right) \wedge \pi$ if $\Phi=\left\{\varphi_{1}, \ldots, \varphi_{n}\right\}$. 
For proving the completeness of $\mathbf{M r L}+$ we need the model that arises naturally from the definition of normal forms and their descriptive nature which was stated in the theorem above. Normal forms as worlds directly convert semantics into syntax, and vice versa.

Definition 5. The triple $M_{n}=\left\langle W_{n}, R_{n}, V_{n}\right\rangle$ is the partial Kripke model such that

- $W_{n}$ is the set of normal forms of degree smaller than or equal to $n$;

- $R_{n}([\pi, \Phi], \alpha) \Leftrightarrow \alpha \in \Phi\left({ }^{5}\right)$

- $V_{n}([\langle P, Q\rangle, \Phi], p)=1 \Leftrightarrow p \in P \& V_{n}([\langle P, Q\rangle, \Phi], p)=0 \Leftrightarrow p \in Q$

$\& V_{n}(\langle P, Q\rangle, p)=1 \Leftrightarrow p \in P \& V_{n}(\langle P, Q\rangle, p)=0 \Leftrightarrow p \in Q$.

Observation 3 . We easily deduce that

- $\theta_{n}\left(M_{n}, \alpha\right)=\alpha$

- $M_{n}, \alpha \models \varphi \Leftrightarrow \alpha \vdash \varphi$ for all $\varphi \in \mathcal{L}_{n}^{\square}$.

The following theorem states the disjunction property that we are looking for, and gives us directly the completeness proof.

THEOREM 2. Every formula of modal depth $n$ is equivalent to a disjunction of normal forms of degree smaller than or equal to $n\left({ }^{6}\right)$.

Corollary. MrL+ is complete with respect to the partial Kripke models.

Proof. Let $\varphi \nvdash \psi$ and let $n$ be the maximum of the modal depths of $\varphi$ and $\psi$. Let $\varphi \equiv \bigvee \Xi$ such that the modal depth of the elements of $\Xi$ are not larger than $n$ (Theorem 2). Clearly $\bigvee \Xi \nvdash \psi$, and so there exists $\xi \in \Xi$ such that $\xi \nvdash \psi\left({ }^{7}\right)$. Observation 3 shows $M_{n}, \xi \models \varphi$ and $M_{n}, \xi \not \psi$, and so $\varphi \not \psi$.

By the finiteness of $M_{n}$ and the completeness we also have decidability for $\mathrm{MrL}+$.

Corollary. MrL+ is decidable.

Because the proof of Theorem 2 needs a lot of inaccessible syntactic treatment, we will outline the argumentation along some lemmas that turn out to be crucial.

LEMMA 1. Let $\Psi$ and $\Phi$ be two finite sets of formulae, with $\Psi \subseteq \Phi$. Then

$$
\square \bigvee \Phi \wedge \bigwedge \diamond \equiv \bigvee_{\Psi \subseteq \Xi \subseteq \Phi}(\square \bigvee \Xi \wedge \bigwedge \diamond \Xi) \equiv \bigvee_{\Psi \subseteq \Xi \subseteq \Phi}[\Xi]
$$

$\left({ }^{5}\right)$ Note that normal forms of degree 0 have no successors, and so $R_{0}=\emptyset$.

( $\left.{ }^{6}\right)$ Remember that the empty disjunction is defined as $\perp$.

$\left(^{7}\right) \xi_{1} \vdash \psi \& \xi_{2} \vdash \psi \Rightarrow \xi_{1} \vee \xi_{2} \vdash \psi$. 
Pr o of. The proof runs by induction on the magnitude of $\Phi / \Psi$. The basic step, $\Phi=\Psi$, is immediate, because the left-hand side of the equation has in this case already the format of the right-hand side (disjunction over the singleton $\{[\Phi]\}$ ).

Let $\#(\Phi / \Psi)=n>0$ and $\Phi=\left\{\varphi_{1}, \ldots, \varphi_{m}, \varphi_{m+1}, \ldots, \varphi_{m+n}\right\}$ and $\Psi=$ $\left\{\varphi_{1}, \ldots, \varphi_{m}\right\}$.

First we observe $\square \bigvee \Phi \vdash \square \bigvee \Psi \bigvee \bigvee \diamond \Phi / \Psi$ (Observation 2). By the propositional rules the following sequence of $\equiv$-equivalences holds:

$$
\begin{aligned}
\square \bigvee \Phi \wedge \bigwedge \diamond \Psi & \equiv(\square \bigvee \Phi \wedge \bigwedge \diamond \Psi) \wedge(\square \bigvee \Psi \vee \bigvee \diamond \Phi / \Psi) \\
& \equiv \bigvee_{j=1}^{n}\left(\square \bigvee \Phi \wedge \bigwedge \diamond\left(\Psi \cup\left\{\varphi_{m+j}\right\}\right)\right) \vee(\square \bigvee \Psi \wedge \bigwedge \diamond \Psi)\left(^{8}\right) \\
& \equiv \bigvee_{j=1}^{n}\left(\bigvee_{\substack{\Psi \subseteq \Xi \subseteq \Phi \\
\varphi_{m+j} \in \Xi}}[\Xi]\right) \vee[\Psi] \quad \text { (by induction hypothesis) } \\
& \equiv \bigvee_{\Psi \subseteq \Xi \subseteq \Phi}[\Xi] .
\end{aligned}
$$

The following lemma needs some formal notation. By $b t(X, Y)$ we mean the set of all bi-total relations in $X \times Y$. Bi-total relations in $X \times Y$ are those relations in $X \times Y$ such that all members of $X$ appear at least once in the first argument, and all members of $Y$ in the second: $R \in b t(X, Y) \Leftrightarrow \forall x \in X \exists y \in Y$ : $\langle x, y\rangle \in R \& \forall y \in Y \exists x \in X:\langle x, y\rangle \in R$. If $R$ is a binary relation, $R^{1}$ is the set of all first arguments of $R: R^{1}=\{x \mid\langle x, y\rangle \in R$ for certain $y\}$. By $R^{2}$ we will refer to the set of all second arguments of $R$. Summarizing, bt $(X, Y)=\left\{R \subseteq X \times Y \mid R^{1}=X, R^{2}=Y\right\}$.

Lemma 2. Let $\Phi$ and $\Psi$ be two finite sets of formulae. Then

$$
\square \bigvee(\Phi \wedge \Psi) \wedge \bigwedge \diamond(\Phi \cup \Psi) \equiv \bigvee_{R \in b t(\Phi, \Psi)}\left(\square \bigvee_{\langle\varphi, \psi\rangle \in R}(\varphi \wedge \psi) \wedge \bigwedge_{\langle\varphi, \psi\rangle \in R} \diamond(\varphi \wedge \psi)\right)
$$

Pr o of. To begin with, note that the following equivalence holds, as a consequence of the preceding lemma.

$$
\begin{aligned}
\square \bigvee(\Phi \wedge \Psi) & \wedge \bigwedge \diamond(\Phi \cup \Psi) \\
& \equiv \bigvee_{R \subseteq \Phi \times \Psi}\left(\square \bigvee_{\langle\varphi, \psi\rangle \in R}(\varphi \wedge \psi) \wedge \bigwedge_{\langle\varphi, \psi\rangle \in R} \diamond(\varphi \wedge \psi) \wedge \bigwedge \diamond(\Phi \cup \Psi)\right) .
\end{aligned}
$$

Let $R$ be a certain relation on $\Phi \times \Psi$ which is not bi-total. This means there exists a $\varphi^{\prime} \in \Phi$ such that $\varphi^{\prime} \notin R^{1}$. Consider the disjunct on the right-hand side of

$\left(^{8}\right)$ By $\wedge / \vee$-distribution, and "modal absorption": $\varphi \vdash \psi \Rightarrow \square \varphi \vdash \square \psi \Rightarrow \square \varphi \wedge \square \psi \equiv \square \varphi$. 
the equivalence stated above, which belongs to this relation $R$. For this particular disjunct the following sequence of derivations holds:

$$
\begin{aligned}
& \square \bigvee_{\langle\varphi, \psi\rangle \in R}(\varphi \wedge \psi) \wedge \bigwedge_{\langle\varphi, \psi\rangle \in R} \diamond(\varphi \wedge \psi) \wedge \bigwedge \diamond(\Phi \cup \Psi) \\
& \vdash \bullet \bigvee_{\langle\varphi, \psi\rangle \in R}(\varphi \wedge \psi) \wedge \bigwedge_{\langle\varphi, \psi\rangle \in R} \diamond(\varphi \wedge \psi) \wedge \diamond \varphi^{\prime} \\
& \equiv \square \bigvee_{\langle\varphi, \psi\rangle \in R}(\varphi \wedge \psi) \wedge \bigwedge_{\langle\varphi, \psi\rangle \in R} \diamond(\varphi \wedge \psi) \wedge \diamond\left(\left(\bigvee_{\langle\varphi, \psi\rangle \in R}(\varphi \wedge \psi)\right) \wedge \varphi^{\prime}\right) \\
& \text { (by } \square \varphi \wedge \diamond \psi \vdash \diamond(\varphi \wedge \psi) \text { ) } \\
& \equiv \square \bigvee_{\langle\varphi, \psi\rangle \in R}(\varphi \wedge \psi) \wedge \bigwedge_{\langle\varphi, \psi\rangle \in R} \diamond(\varphi \wedge \psi) \wedge\left(\bigvee_{\langle\varphi, \psi\rangle \in R}\left(\diamond\left(\varphi \wedge \psi \wedge \varphi^{\prime}\right)\right)\right) \\
& \vdash \text { • } \bigvee_{\langle\varphi, \psi\rangle \in R}(\varphi \wedge \psi) \wedge \bigwedge_{\langle\varphi, \psi\rangle \in R} \diamond(\varphi \wedge \psi) \wedge\left(\bigvee_{\psi^{\prime} \in R^{2}}\left(\diamond\left(\varphi^{\prime} \wedge \psi^{\prime}\right)\right)\right) \\
& \equiv \bigvee_{\psi^{\prime} \in R^{2}}\left(\square\left(\bigvee_{\langle\varphi, \psi\rangle \in R}(\varphi \wedge \psi) \vee\left(\varphi^{\prime} \wedge \psi^{\prime}\right)\right) \wedge \bigwedge_{\langle\varphi, \psi\rangle \in R} \diamond(\varphi \wedge \psi) \wedge \diamond\left(\varphi^{\prime} \wedge \psi^{\prime}\right)\right) .
\end{aligned}
$$

From the derivation above we learn that the $R$-disjunct with $\varphi^{\prime} \notin R^{1}$ entails a disjunct of other disjuncts appearing in the right-hand side of original equation (1), which corresponds to relations in $\Phi \times \Psi$ which have $\varphi^{\prime}$ as a first argument. The same holds for relations $R^{\prime}$ in $\Phi \times \Psi$ with $R^{\prime 2} \neq \Psi$. Henceforth we may conclude that the equivalence (1) holds also if we range only over bi-total relations in $\Phi \times \Psi$ $\left(\varphi^{\prime} \notin R^{1}\right.$ was arbitrarily chosen, and $\left.\varphi \vdash \psi \Leftrightarrow \psi \equiv \psi \vee \varphi\right)$. So,

$$
\begin{aligned}
\square \bigvee(\Phi \wedge \Psi) & \wedge \curlywedge(\Phi \cup \Psi) \\
& \equiv \bigvee_{R \in b t(\Phi \times \Psi)}\left(\square \bigvee_{\langle\varphi, \psi\rangle \in R}(\varphi \wedge \psi) \wedge \bigwedge_{\langle\varphi, \psi\rangle \in R} \diamond(\varphi \wedge \psi) \wedge \bigwedge \diamond(\Phi \cup \Psi)\right) .
\end{aligned}
$$

Because $\diamond(\varphi \wedge \psi) \vdash \diamond \varphi$ and $\diamond(\varphi \wedge \psi) \vdash \diamond \psi$ and because all $\Phi$ and $\Psi$ are fully present in the first $\diamond$-conjuncts, the last $\diamond$-conjuncts get all absorbed by the first $\diamond$-conjuncts $(\varphi \vdash \psi \Leftrightarrow \varphi \equiv \varphi \wedge \psi)$. We end up with the result that we were looking for:

$$
\square \bigvee(\Phi \wedge \Psi) \wedge \bigwedge \diamond(\Phi \cup \Psi) \equiv \bigvee_{R \in b t(\Phi, \Psi)}\left(\square \bigvee_{\langle\varphi, \psi\rangle \in R}(\varphi \wedge \psi) \wedge \bigwedge_{\langle\varphi, \psi\rangle \in R} \diamond(\varphi \wedge \psi)\right)
$$

LEMma 3. Every conjunct of two normal forms of degree $n$ is equivalent to a disjunction over a finite set of normal forms of degree $n$.

P r o of. By induction on the degree of normal forms. The basic propositional case is directly obtained. In this case we end up with either one disjunct, or zero, which is defined as $\perp$. 
Let $[\pi, \Phi]$ and $[\varrho, \Psi]$ be two normal forms of degree $n+1(n \geq 0)$. By Lemma 2 we immediately have the following equivalence:

$$
\begin{aligned}
{[\pi, \Phi] \wedge[\varrho, \Psi] } & =\square \bigvee \Phi \wedge \bigwedge \diamond \Phi \wedge \pi \wedge \square \bigvee \Psi \wedge \bigwedge \diamond \Psi \wedge \varrho \\
& \equiv \square(\bigvee \Phi \wedge \bigvee \Psi) \wedge \bigwedge \diamond(\Phi \cup \Psi) \wedge \pi \wedge \varrho \\
& \equiv \square(\bigvee(\Phi \wedge \Psi)) \wedge \bigwedge \diamond(\Phi \cup \Psi) \wedge \pi \wedge \varrho \\
& \equiv \bigvee_{R \in b t(\Phi, \Psi)}\left(\square \bigvee_{\langle\varphi, \psi\rangle \in R}(\varphi \wedge \psi) \wedge \bigwedge_{\langle\varphi, \psi\rangle \in R} \diamond(\varphi \wedge \psi)\right) \wedge \pi \wedge \varrho .
\end{aligned}
$$

The induction hypothesis gives us the disjunction property for the pairs $\varphi \wedge \psi$ (being normal forms of degree $n$ ). This disjunction will be referred to as $\bigvee \Xi_{\varphi, \psi}$, where every member of this disjunct is a normal form of degree $n$.

(2) $[\pi, \Phi] \wedge[\varrho, \Psi]$

$$
\begin{aligned}
& \equiv \bigvee_{R \in b t(\Phi, \Psi)}\left(\left(\square \bigvee_{\langle\varphi, \psi\rangle \in R}\left(\bigvee \Xi_{\varphi, \psi}\right)\right) \wedge \bigwedge_{\langle\varphi, \psi\rangle \in R} \diamond\left(\bigvee \Xi_{\varphi, \psi}\right)\right) \wedge \pi \wedge \varrho \\
& \equiv \bigvee_{R \in b t(\Phi, \Psi)}\left(\left(\square \bigvee_{\langle\varphi, \psi\rangle \in R}\left(\bigvee \Xi_{\varphi, \psi}\right)\right) \wedge \bigwedge_{\langle\varphi, \psi\rangle \in R}\left(\bigvee \diamond\left(\Xi_{\varphi, \psi}\right)\right)\right) \wedge \pi \wedge \varrho .
\end{aligned}
$$

Before moving on, let us reduce the syntax somewhat. We first forget about the propositional part $\pi \wedge \varrho$, because it does not influence the line of argument. The general form of the disjuncts in (2) is now

$$
\square\left(\bigvee_{i=1}^{m} \bigvee \Phi_{i}\right) \wedge \bigwedge_{i=1}^{m}\left(\bigvee \diamond \Phi_{i}\right)\left({ }^{9}\right)
$$

First note that by distribution of $\wedge$ over $\vee$ we obtain $\bigwedge_{i=1}^{m} \bigvee \Phi_{i} \equiv \bigvee_{\mathrm{c} \in \mathcal{C}} \bigwedge_{i=1}^{m} \mathrm{c}\left(\Phi_{i}\right)$ where $\mathcal{C}$ is the set of all distinct choice functions that select for every $i$ an element of $\Phi_{i}$. And so,

$$
\begin{aligned}
\square\left(\bigvee_{i=1}^{m} \bigvee \Phi_{i}\right) \wedge \bigwedge_{i=1}^{m}\left(\bigvee \diamond \Phi_{i}\right) & \equiv \square\left(\bigvee_{i=1}^{m} \bigvee \Phi_{i}\right) \wedge \bigvee_{\mathrm{c} \in \mathcal{C}} \bigwedge_{i=1}^{m} \diamond \mathrm{c}\left(\Phi_{i}\right) \\
& \equiv \bigvee_{\mathrm{c} \in \mathcal{C}}\left(\square \bigvee_{i=1}^{m} \bigvee \Phi_{i}\right) \wedge \bigwedge_{i=1}^{m} \diamond \mathrm{c}\left(\Phi_{i}\right)
\end{aligned}
$$

All the disjuncts in the last formula are of the form $\square \bigvee \Phi \wedge \wedge \diamond \Psi$ with $\Psi \subseteq \Phi$. Application of Lemma 1, and the fact that all formulae in $\Phi_{i}$ are normal forms $\left({ }^{10}\right)$, show us that every disjunct in the formula is equivalent to a disjunction of normal forms. This ends the proof of the lemma, having a disjunction of a disjunction of

$\left({ }^{9}\right)$ The empty cases $m=0$ and $\Phi_{i}=\emptyset$ are left to the reader.

$\left({ }^{10}\right)$ Note that also $\mathrm{c}\left(\Phi_{i}\right) \in \Phi_{i}$. 
a disjunction of normal forms. Readers who are interested in the final result may consider the final equivalence:

$$
[\pi, \Phi] \wedge[\varrho, \Psi] \equiv \bigvee_{R \in b t(\Phi, \Psi)} \bigvee_{\mathbf{c} \in \mathcal{C}} \bigvee_{\mathfrak{c}(\Theta) \subseteq \Xi \subseteq \Theta}[\pi \wedge \varrho, \Xi]\left({ }^{11}\right),
$$

with $\Theta=\bigcup \Xi_{\varphi, \psi}, \bigvee \Xi_{\varphi, \psi} \equiv \varphi \wedge \psi$ with all members of $\Xi_{\varphi, \psi}$ being normal forms of degree $n$ (induction) and $\mathcal{C}$ being the collection of all distinct choice functions that pick an element of $\Xi_{\varphi, \psi}$ for every pair $\varphi \in \Phi, \psi \in \Psi$, and $\mathrm{c}(\Theta)=\left\{\mathrm{c}\left(\Xi_{\varphi, \psi}\right) \mid\right.$ $\varphi \in \Phi, \psi \in \Psi\}$.

We have proved that conjoining two normal forms of degree $n$ amounts to a disjunct of normal forms of degree $n$. The following lemmas $(4,5)$ guarantee that we are in fact independent of the degree of those normal forms. This will be forced by proving that every normal form of degree $n$ is equivalent to a disjunction of normal forms of arbitrary larger degree $m(\geq n)$. First we need the disjunction of normal forms that contain no information, that is, they are theorems in $\mathbf{M r L}+$.

Lemma 4. Let $T_{0}=\{\top\}$ and $T_{n}=\left\{[\Phi] \mid \Phi \subseteq T_{n-1}\right\}$. The disjunction over such a set $T_{n}$ is a theorem in $\mathbf{M r L +}$

$$
\forall n \in \mathbb{N}: \vdash \bigvee T_{n}
$$

Proof. By induction on $n$. The case $n=0$ is even an axiom (see Def. 3 ). Let $n>0$. The induction hypothesis gives us $\vdash \bigvee T_{n-1}$. The last inference rule in $\mathbf{M r L}+$ (Def. 3) yields $\vdash$ $\bigvee T_{n-1}$, and by Lemma 1 this can be rewritten as $\vdash \bigvee_{\Phi \subseteq T_{n-1}}[\Phi]$. This last formula is, by definition of $T_{n}$, the same as $\bigvee T_{n}$.

LEMma 5. Every normal form of degree $n$ is equivalent to a disjunction of normal forms of degree $n+m(m \in \mathbb{N})$.

Proof. By induction on $n$. For the case $n=0$, we take $\pi \equiv \bigvee_{\Phi \subseteq T_{m-1}}[\pi, \Phi]$, which can be proved by a straightforward accommodation of the proof of Lemma 4.

Let $n>0$. Suppose $[\pi, \Phi]$ is a normal form of degree $n$. We may apply the induction hypothesis to every member of $\Phi$, being a normal form of degree $n-1$. We have

$$
\forall \varphi \in \Phi: \varphi \equiv \bigvee \Xi_{\varphi}
$$

with every member of $\Xi_{\varphi}$ being a normal form of degree $(n-1)+m$. Therefore, $[\pi, \Phi]$ must be equivalent to a disjunction of normal forms of degree $n+m$, by

$\left.{ }^{(11}\right) \pi \wedge \varrho$ does not have to be a normal form of degree 0 . If $\pi \wedge \varrho \equiv \perp$ we have the empty disjunct, and therefore the same result. In the other case we take $\left[\left\langle P \cup P^{\prime}, Q \cup Q^{\prime}\right\rangle, \Xi\right]$ for the disjuncts with $\pi=\langle P, Q\rangle$ and $\varrho=\left\langle P^{\prime}, Q^{\prime}\right\rangle$. 
the following derivation:

$$
\begin{aligned}
{[\pi, \Phi] } & =\square \bigvee \wedge \wedge \diamond \Phi \wedge \pi \\
& \equiv \square \bigvee_{\varphi \in \Phi} \bigvee \Xi_{\varphi} \wedge \bigwedge_{\varphi \in \Phi} \diamond \bigvee \Xi_{\varphi} \wedge \pi \\
& \equiv \square \bigvee_{\varphi \in \Phi} \bigvee \Xi_{\varphi} \wedge \bigwedge_{\varphi \in \Phi}\left(\bigvee \diamond \Xi_{\varphi}\right) \wedge \pi \\
& \equiv \bigvee_{c \in \mathcal{C}}\left(\square \bigvee_{\varphi \in \Phi} \bigvee \Xi_{\varphi} \wedge \bigwedge \diamond c\left(\Xi_{\varphi}\right) \wedge \pi\right)\left({ }^{12}\right) \\
& \equiv \bigvee_{c \in \mathcal{C}} \bigvee_{c}[\pi, \Xi] . \text { - }
\end{aligned}
$$

COROLlaRY. The conjunction of two normal forms is equivalent to a disjunction of normal forms.

At last we have gathered enough syntactic equipment for proving the central theorem.

Proof of Theorem 2. By induction on the modal depth of formulae. In the basic propositional case, we immediately find the desired result by propositional reasoning (the principle of the excluded middle is not necessary here). Let $\varphi$ be an arbitrary formula with modal depth $n \geq 1$. To begin with, we translate $\varphi$ by using the propositional part of $\mathbf{M r L}+$ as for $n=0$, into a "propositional disjunctive normal form", that is, a disjunction over conjunctions, such that the resulting conjuncts are only of the form $\square \psi \diamond \diamond \psi$ (with modal depth of $\psi$ being smaller than $n), \neg p$ or $p(p \in \mathbb{P})$. The propositional cases are left, because they will not bother us. Because of the corollary stated above, we only have to prove that $\square \bigvee \Phi$ and $\diamond \bigvee \Phi$ with $\Phi$ being normal forms of degree smaller than $n$ are equivalent to a disjunction of normal forms. The first case follows immediately from Lemma 1, only the $\diamond$-case needs some clarification. This is done with the help of Lemmas 4, 5 and Lemma 1 . First, we may put $\bigvee$ outside the $\diamond$-scope:

$$
\diamond \bigvee \Phi \equiv \bigvee \diamond \Phi
$$

Let $\diamond \xi$ be a disjunct in the last formula, and let $m<n$ be the modal depth of $\xi$. Since $\vdash$ $\bigvee T_{m}$ the following equivalences hold:

$$
\diamond \xi \equiv \square\left(\bigvee T_{m} \vee \xi\right) \wedge \diamond \xi \equiv \bigvee_{\xi \in \Xi \subseteq T_{m} \cup\{\xi\}}[\Xi]
$$

Having used the induction hypothesis we arrived at an equivalent $\varphi \equiv \bigvee \wedge \bigvee \Phi_{1}$ $\wedge /$-distribution reformats this into $\bigvee \wedge \Phi_{2}$, and finally we obtain $\varphi \equiv \bigvee \Phi_{3}$ by

$\left({ }^{12}\right)$ With $\mathcal{C}$ being the class of distinct choice functions which pick for any $\varphi \in \Phi$ an element of $\Xi_{\varphi}$. 
Lemma 3 and its generalization summarized in the corollary above $\left(\Phi_{i}\right.$ 's being all sets of normal forms of degree $n$ ).

Conclusions and implications. Normal forms as introduced in modal logic in [Fin] have been transposed to partial modal logic, by giving them a slightly different format. We could state that the enterprise has been a relative success. The meta-theoretical results which were obtained in [Fin] were adopted, but the road has been disproportionately troublesome. This is due to the disturbing role of the partial negation. Semantically speaking, negation is no longer interpreted as the technically more accessible complement, but as a so-called quasi-complement [Ras], that is, a complement that obeys de Morgan equations, and coherence $(p \wedge \neg p=\perp)$, but which does not necessarily behave according to the law of the excluded middle $(p \vee \neg p=\top)\left({ }^{13}\right)$.

A very important implication of the results above is that we have direct access to the class of formulae that have precisely one minimal interpretation. These are precisely the normal forms modulo $\equiv$. This minimality is guaranteed by the embedding of partial Kripke models into the model of all normal forms.

TheOREM 3. Let $\varphi$ be a formula of modal depth $n . \varphi$ is equivalent to a normal form of degree $n$ iff $\varphi$ has a minimal interpretation, that is, there exists a world $w$ in a model $M=\langle W, R, V\rangle$ such that $M, w \models \psi$ iff $\varphi \models \psi(\varphi \vdash \psi)$ for all formulae $\psi \in \mathcal{L}_{n}^{\square}\left({ }^{14}\right)$.

Proof. Let $\varphi \equiv \varphi^{\prime}$ with $\varphi^{\prime}$ being a normal form of degree not larger than $n$. Clearly $M_{n}, \varphi^{\prime} \vDash \psi \Leftrightarrow \varphi \models \psi$ for all formulae $\psi$ with modal depth not larger than $n$.

This kind of minimality is relevant if we want to find minimal representations for propositional attitudes that we would like to model in partial modal logic.

The informational (theoretical) order $\models$ on normal forms can also be determined structurally, in a relatively easy way.

O bservation 4 . Let $\langle P, Q\rangle$ and $\left\langle P^{\prime}, Q^{\prime}\right\rangle$ be two normal forms of degree 0 . We define

$$
\langle P, Q\rangle \leq_{0}\left\langle P^{\prime}, Q^{\prime}\right\rangle \Leftrightarrow P \subseteq P^{\prime} \& Q \subseteq Q^{\prime} .
$$

Let $[\pi, \Phi]$ and $[\varrho, \Psi]$ be two normal forms of degree $n$. We order them by the following recursive definition:

$$
\begin{aligned}
{[\pi, \Phi] \leq_{n}[\varrho, \Psi] \Leftrightarrow } & \pi \leq_{0} \varrho \& \\
& \forall \varphi \in \Phi \exists \psi \in \Psi: \varphi \leq_{n-1} \psi \& \forall \psi \in \Psi \exists \varphi \in \Phi: \varphi \leq_{n-1} \psi .
\end{aligned}
$$

$\left.{ }^{13}\right)$ A way to overcome this problem is to take a partial accessibility in the Kripke frames as well [Mus]. We avoided this approach because our intuitions about the role of possible worlds in interpreting propositional attitudes do not fit with such an interpretation. Besides that, the resulting logic is of course different, due to the less restricted interplay of the modal operators.

$\left.{ }^{(14}\right) \theta_{n}(M, w) \equiv \varphi$. 
It turns out that this structural order precisely follows the theoretical order:

$$
[\varrho, \Psi] \vDash[\pi, \Phi] \Leftrightarrow[\varrho, \Psi] \vdash[\pi, \Phi] \Leftrightarrow[\pi, \Phi] \leq_{n}[\varrho, \Psi] .
$$

The pre-order $\leq_{n}$ intuitively tells us that a world $w$ is theoretically or informationally richer than another world $w^{\prime}$ if both the objective propositional part of $w$ is larger $\left(\geq_{0}\right)$ than $w^{\prime}$, and all successors of $w$ are larger $\left(\geq_{n-1}\right)$ than some successor of $w^{\prime}$ and all successors of $w^{\prime}$ are smaller $\left(\leq_{n-1}\right)$ than some successor of $w$. The first relation preserves objective information, the second takes care of 口- and the third of $\diamond$-preservation. We will not give a proof of this observation. It can simply be done by induction on the degree of normal forms. In [Jas] one will find an elaborate exposition of how to compose minimal interpretations in partial modal logic. In this paper partial modal structures have been used, after the idea of modal structures in $[\mathrm{FaV}]$, which is just a syntactical variant of the normal forms in [Fin].

Another issue that we like to point at is our definition of consequence. In partial logic one also finds the so-called double barreled consequence relation [Bla]. $\psi$ is a double barreled consequence of $\varphi$ iff all models $M$ and all worlds $w$ that support $\varphi$ also support $\psi$, and all such pairs that reject $\psi$ also reject $\varphi$ $(M, w \neq \psi \Rightarrow M, w \neq \varphi)$. This can be encoded in our system by $\varphi \models \psi$ \& $\neg \psi \models \neg \varphi$. This consequence relation restores contraposition. The resulting logic is $\mathbf{M r L}+$, without the ex falso rules $\varphi \wedge \neg \varphi \vdash \perp$ and $\diamond(\varphi \wedge \neg \varphi) \vdash \perp$. A complete axiomatization can be given by adding weaker versions: $\varphi \wedge \neg \varphi \vdash \psi \vee \neg \psi$ and $\diamond(\varphi \wedge \neg \varphi) \vdash \square(\psi \vee \neg \psi)$ [Thi]. Besides this double barreled definition, there is more freedom here [Thi] (e.g. $M, w \models \varphi \Rightarrow M, w \nexists \psi$ ). But still, in these approaches normal forms can be used as the semantic unit for extracting meta-theoretical results for different consequence relations. Normal forms are the precise syntactic representation of the $\vDash$ and $\neq$ behavior of worlds. This insight makes normal forms a useful tool in partial modal logic.

\section{References}

[BaP] J. Barwise and J. Perry, Situations and Attitudes, MIT Press, Cambridge, MA, 1983.

[Bla] S. Blamey, Partial logic, in: Handbook of Philosophical Logic, D. Gabbay and F. Guenthner (eds.), \#III, Reidel, Dordrecht 1986, 1-70.

[FaH $] \quad$ R. Fagin and J. Y. Halpern, Belief, awareness and limited reasoning, in : Proc. Ninth International Joint Conference on Artificial Intelligence, Morgan Kaufmann, Los Altos 1985, 491-501.

[FaV] R. Fagin and M. Y. Vardi, An internal semantics for modal logic: preliminary report, CSLI Research Notes \#85-25, Stanford Univ., 1985.

[Fin] K. Fine, Normal forms in modal logic, Notre Dame J. Formal Logic 16 (1975), 229-237.

[Jas] J. O. M. Jaspars, Theoretical circumscription in partial modal logic, in: Logics in AI, JELIA '90, J. van Eijck (ed.), Lecture Notes in Artificial Intelligence 478, Springer, Heidelberg 1991, 303-318.

[Kam] H. Kamp, A scenic tour through the land of naked infinitives, manuscript, 1983.

[Mus] R. A. Muskens, Meaning and partiality, Ph.D. thesis, Univ. of Amsterdam, 1989. 
[Ras] H. Rasiowa, An Algebraic Approach to Non-Classical Logics, North-Holland, Amsterdam 1974.

[Thi] E. G. C. Thijsse, Partial propositional and modal logic; the overall theory, in: Proc. Seventh Amsterdam Colloquium, M. Stokhof and L. Torenvliet (eds.), ITLI, Amsterdam 1990, 555-579. 